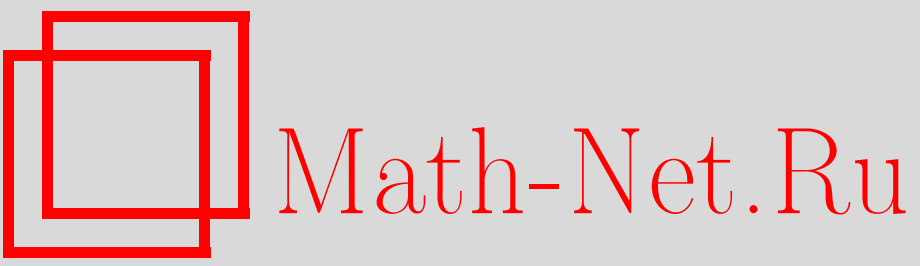

Г. А. Чечкин, Расщепление кратного собственного значения в задаче о концентрированных массах, УМН, 2004, том 59, выпуск 4, 205-206

DOI: https://doi.org/10.4213/rm769

Использование Общероссийского математического портала Math-Net.Ru подразумевает, что вы прочитали и согласны с пользовательским соглашением

http://www.mathnet.ru/rus/agreement

Параметры загрузки:

IP : 35.173 .219 .149

26 апреля 2023 г., 13:58:12 


\title{
РАСЩЕПЛЕНИЕ КРАТНОГО СОБСТВЕННОГО ЗНАЧЕНИЯ В ЗАДАЧЕ О КОНЦЕНТРИРОВАННЫХ МАССАХ
}

\author{
Г.А. ЧЕчкин
}

Пусть $\Omega$ - область в $\mathbb{R}^{2}$, лежащая в верхней полуплоскости, граница которой является кусочно гладкой и состоит из нескольких частей: $\partial \Omega=\Gamma_{1} \cup \Gamma_{2} \cup \Gamma_{3} \cup \Gamma_{4}$, при этом $\Gamma_{4}=\Gamma_{\varepsilon} \cup \gamma_{\varepsilon}$, где $\Gamma_{4}$ - интервал $\left(-\frac{\pi}{2}, \frac{\pi}{2}\right)$ на оси абсцисс, а $\Gamma_{2}$ и $\Gamma_{3}$ принадлежат прямым $x_{1}=-\frac{\pi}{2}$ и $x_{1}=\frac{\pi}{2}$ соответственно. Здесь $\varepsilon=\frac{1}{2 \mathcal{N}+1}-$ малшій параметр, $\mathscr{N}$ - натуральное число, $\mathscr{N} \gg 1$. Опишем подробнее мелкомасштабную структуру $\Gamma_{4}$. Пусть $\gamma=\left\{\xi:-a<\xi_{1}<a, \xi_{2}=0\right\}, \Gamma=\{\xi$ : $\left.-\frac{\pi}{2}<\xi_{1}<-a, a<\xi_{1}<\frac{\pi}{2}, \xi_{2}=0\right\}$ в переменных $\xi=\frac{x}{\varepsilon}$, при этом $0<a<\frac{\pi}{2}$. Обозначим $\gamma_{\varepsilon}=\left\{x \in \Gamma_{4}: \varepsilon^{-1}\left(x_{1}-j, 0\right) \in \gamma, j \in \mathbb{Z}\right\}, \Gamma_{\varepsilon}=\Gamma_{4} \backslash \gamma_{\varepsilon}$. Обозначим $\Pi=\left\{\xi:-\frac{\pi}{2}<\xi_{1}<\frac{\pi}{2}\right.$, $\left.\xi_{2}>0\right\}, B=\left\{\xi: \xi_{1}^{2}+\xi_{2}^{2}<a^{2}, \xi_{2}>0\right\}, B_{\varepsilon}^{j}=\left\{x \in \Omega: \varepsilon^{-1}\left(x_{1}-j, x_{2}\right) \in B\right\}, j \in \mathbb{Z}$, $B_{\varepsilon}=\bigcup B_{\varepsilon}^{j}$.

Рассмотрим задачу

$$
-\Delta u_{\varepsilon}=\lambda_{\varepsilon} \rho_{\varepsilon} u_{\varepsilon} \text { при } x \in \Omega, \quad u_{\varepsilon}=0 \text { при } x \in \gamma_{\varepsilon}, \quad \frac{\partial u_{\varepsilon}}{\partial \nu}=0 \text { при } x \in \Gamma_{\varepsilon} \cup \Gamma_{1} \cup \Gamma_{2} \cup \Gamma_{3},
$$

где $\rho_{\varepsilon}(x)$ равна 1 в $\Omega \backslash \bar{B}_{\varepsilon}$ и равна $(a \varepsilon)^{-m}$ в $B_{\varepsilon}, 0<m<2$. Будем называть множества $B_{\varepsilon}^{j}$ концентриров анными массами.

Можно показать (см., например, [1], а также [2]), что задача

$$
-\Delta u_{0}=\lambda u_{0} \text { при } x \in \Omega, \quad u_{0}=0 \text { при } x \in \Gamma_{4}, \quad \frac{\partial u_{0}}{\partial \nu}=0 \text { при } x \in \Gamma_{1} \cup \Gamma_{2} \cup \Gamma_{3}
$$

является предельной для задачи $(1)$ при $\varepsilon \rightarrow 0$. В частности, к собственному значению $\lambda_{0}$ кратности $N$ задачи (2) сходятся $N$ собственных значений (с учетом совокупной кратности) задачи (1). Целью настоящей работы является построение асимптотики собственных значений задачи (1), сходящихся к двукратному собственному значению $\lambda_{0}$ задачи (2).

Для построения асимптотики воспользуемся методом согласования асимптотических разложений (см. [3], [4]).

Обозначим две собственные функции задачи (2), соответствующие собственному значению $\lambda_{0}$, через $u_{0}^{(1)}$ и $u_{0}^{(2)}$. Считаем, что они нормированы в $L_{2}(\Omega)$. Обозначим $\alpha_{0}^{(\theta)}\left(x_{1}\right)=\left.\frac{\partial u_{0}^{(\theta)}}{\partial x_{2}}\right|_{x_{2}=0}$. 3 десь и далее $\theta=1,2$. Легко видеть, что эти функции можно выбрать так, чтобы они удовлетво-

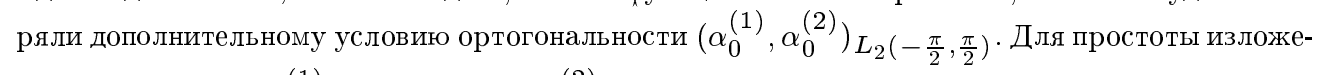
ния считаем, что $\left\|\alpha_{0}^{(1)}\right\|_{L_{2}\left(-\frac{\pi}{2}, \frac{\pi}{2}\right)} \neq\left\|\alpha_{0}^{(2)}\right\|_{L_{2}\left(-\frac{\pi}{2}, \frac{\pi}{2}\right)}$.

Внешнее разложение (вне малой окрестности $\Gamma_{4}$ ) строится в виде

$$
u_{\varepsilon}^{(\theta)}(x)=u_{0}^{(\theta)}(x)+\varepsilon u_{1}^{(\theta)}(x)+\cdots,
$$

внутреннее разложение (в малой окрестности $\Gamma_{4}$ ) строится в виде

$$
u_{\varepsilon}^{(\theta)}(x)=\varepsilon v_{1}^{(\theta)}\left(\frac{x}{\varepsilon} ; x_{1}\right)+\cdots
$$

а разложение собственных значений -

$$
\lambda_{\varepsilon}^{(\theta)}=\lambda_{0}+\varepsilon \lambda_{1}^{(\theta)} \cdots
$$

Работа выполнена при частичной поддержке Российского фонда фундаментальных исследований (грант № 02-01-00693). 
Краевая задача для коэффицциента $v_{1}^{(\theta)}\left(\xi ; x_{1}\right)$ имеет вид

$$
\left\{\begin{array}{l}
\Delta_{\xi} v_{1}^{(\theta)}=0 \text { в П, } \\
v_{1}^{(\theta)}=0 \text { на } \gamma, \\
\frac{\partial v_{1}^{(\theta)}}{\partial \xi_{2}}=0 \text { на } \Gamma, \\
\frac{\partial v_{1}^{(\theta)}}{\partial \xi_{1}}\left(\xi ; \pm \frac{\pi}{2}\right)=0 \text { при } \xi_{1}= \pm \frac{\pi}{2}, \\
v_{1}^{(\theta)} \sim \alpha_{0}^{(\theta)} \xi_{2} \text { при } \xi_{2} \rightarrow+\infty .
\end{array}\right.
$$

Решение задачи (6) существует и имеет асимптотику при $\xi_{2} \rightarrow+\infty$ :

$$
v_{1}^{(\theta)}(\xi)=\alpha_{0}^{(\theta)}\left(x_{1}\right)\left(\xi_{2}-\ln \sin a\right)+O\left(e^{-2 \xi_{2}}\right) .
$$

Переписьвая асимптотику $\varepsilon v_{1}^{(\theta)}$ во внешних переменных, в силу согласования асимптотических разложений получаем краевую задачу для $u_{1}^{(\theta)}$ :

$$
\left\{\begin{array}{l}
-\Delta u_{1}^{(\theta)}=\lambda_{0} u_{1}^{(\theta)}+\lambda_{1}^{(\theta)} u_{0}^{(\theta)} \text { в } \Omega, \\
u_{1}^{(\theta)}=-\alpha_{0}^{(\theta)} \ln \sin a \text { на } \Gamma_{4}, \\
\frac{\partial u_{1}^{(\theta)}}{\partial \nu}(x)=0 \text { на } \Gamma_{1} \cup \Gamma_{2} \cup \Gamma_{3} .
\end{array}\right.
$$

Из условия разрешимости задачи (8) определяется поправочный член $\lambda_{1}^{(\theta)}$ в разложении (5) по формуле

$$
\lambda_{1}^{(\theta)}=\ln \sin a \int_{-\frac{\pi}{2}}^{\frac{\pi}{2}}\left(\alpha_{0}^{(\theta)}\left(x_{1}\right)\right)^{2} d x_{1} .
$$

Случай простого собственного значения см. в [5]. Отметим также, что дополнителнное условие ортогональности ранее возникало в [6] для задачи с частой сменой типа граничного условия.

\section{СПИСОК ЛИТЕРАТУРЫ}

[1] Г. А. Чечкин // Краевые задачи для неклассических уравнений в частных производных. Новосибирск: ИМ СОАН СССР, 1989. С. 197-200. [2] О. А. Олейник, Г. А. Иосифьян, А. С. Шамаев. Математические задачи теории сильно неоднородных упругих сред. М.: Изд-во МГУ, 1990. [3] А. М. Ильин. Согласование асимптотических разложений решений краевых задач. М.: Наука, 1989. [4] Р. Р. Гадыльшин // Алгебра и анализ. 1998. Т. 10. № 1. С. 3-19. [5] G.A. Chechkin. On vibration of partially fastened membrane with many "light" concentrated masses on the boundary // C. R. Acad. Sci. Paris Sér. IIb. 2004. V. 332 (to appear). [6] Д.И. Борисов. Асимптотики и оценки собственных элементов лапласиана с частой непериодической сменой граничных условий // Изв. РАН. Сер. матем. 2003. Т. 67. №6. С. 23-70.

Московский государственньй

Принято редколлегией университет им. М.В. Ломоносова 24.05 .2004

E-mail: chechkin@mech.math.msu.su 